\title{
MIXED RADIAL BLASCHKE-MINKOWSKI HOMOMORPHISMS AND COMPARISON OF VOLUMES
}

\author{
LIJUAN LIU
}

Abstract. In this paper we consider a Busemann-Petty type problem for mixed radial BlaschkeMinkowski homomorphisms.

Mathematics subject classification (2010): 52A40, 52A20.

Keywords and phrases: Mixed radial Blaschke-Minkowski homomorphisms, mixed dual volume.

\section{REFERENCES}

[1] S. Alesker, A. Bernig, F. E. Schuster, Harmonic Analysis of Translation Invariant Valuations, Geom. Funct. Anal. 21 (2011), 751-773.

[2] K. BALL, Volume in terms of concurrent cross-sections, Pacific J. Math. 3 (1953), 1-12.

[3] R. J. GARDNER, Intersection bodies and the Busemann-Petty problem, Trans. Amer. math. Soc. 342 (1994), 435-445.

[4] R. J. Gardner, A positive answer to the Busemann-Petty problem in three dimensions, Ann. Math. 140 (1994), 435-447.

[5] R. J. Gardner, A. Koldobsky, And T. Schlumprecht, An analytic solution of the BusemannPetty problem on sections of convex bodies, Ann. Math. 149 (1999), 691-703.

[6] E. Grinberg, G. Zhang, Convolutions, transforms and convex bodies, Proc. London Math. Soc. 78 (1999), 77-115.

[7] C. HaberL, $L_{p}$ intersection bodies, Adv. Math. 217 (2008), 2599-2624.

[8] C. HABERL, Star body valued valuations, Indiana Univ. Math. J. 58 (2009), 2253-2276.

[9] C. Haberl, F. E. Schuster, Genneral $L_{p}$ affine isoperimetric inequalities, J. Differential Geom. 83 (2009), 1-26.

[10] N. J. Kalton, A. Koldobsky, Intersection bodies and L p$_{p}$-spaces, Adv. Math. 196 (2005), 257275 .

[11] M. KIDERLEN, Blaschke- and Minkowski-endomorphisms of convex bodies, Trans. Amer. Math. Soc. 358 (2006), 5539-5564.

[12] D. A. KLAIn, Star valuations and dual mixed volumes, Adv. Math. 121 (1996), 80-101.

[13] A. Koldobs Ky, Intersection bodies, positive definite distributions, and the Busemann-Petty problem, Amer. J. Math. 120 (1998), 827-840.

[14] A. Koldobsky, A functional analytic approach to intersection bodies, Geom. Funct. Anal. 10 (2000), 1507-1526.

[15] A. Koldobsky, Fourier Analysis in Convex Geometry, Math. Surveys Monogr., vol. 116, Amer. Math. Soc., 2005.

[16] M. Ludwig, Projection bodies and valuations, Adv. Math. 172, 2 (2002), 158-168.

[17] M. Ludwig, Minkowski valuations, Trans. Amer. Math. Soc. 357, 10 (2005), 4191-4213.

[18] M. Ludwig, Intersection bodies and valuations, Amer. J. Math. 128, 6 (2006), 1409-1428.

[19] E. Lutwak, Dual mixed volumes, Pacific J. Math. 58 (1975), 531-538.

[20] E. LuTwAK, Intersection bodies and dual mixed volumes, Adv. Math. 71 (1988), 232-261.

[21] P. McMullen, R. Schneider, Valuations on convex Bodies, in: P. M. Gruber, J. M. Wills (Eds.), Convexity and Its Applications, Birkhäuser, Basel, 1983, pp. 170-247.

[22] R. Schneider, F. E. Schuster, Rotation equivariant Minkowski valuations, Int. Math. Res. Not. 2006, Art. ID 72894, 20 pp. 
[23] F. E. SChUSTER, Volume inequalities and additive maps of convex bodies, Mathematika 53, 2 (2006), 211-234 (2007).

[24] F. E. SCHUSTER, Convolutions and multiplier transformations of convex bodies, Trans. Amer. Math. Soc. 359, 11 (2007), 5567-5591.

[25] F. E. SChUSTER, Valuations and Busemann-Petty type problems, Adv. Math. 219, 1 (2008), 344-368.

[26] F. E. Schuster, Crofton measures and Minkowski valuations, Duke Math. J. 154, 1 (2010), 1-30.

[27] W. WANG, L. J. LiU AND B. W. HE, $L_{p}$ radial Minkowski homomorphisms, Taiwan J. Math 15, 3 (2011), 1183-1199.

[28] G. Zhang, Centered bodies and dual mixed volumes, Trans. Amer. Math. Soc. 345 (1994), 777-801.

[29] G. Zhang, A positive answer to the Busemann-Petty problem in four dimensions, Ann. Math. 149 (1999), 535-543.

[30] C. J. ZhaO, On Blaschke-Minkowski homomorphisms, Geom. Dedicata 149 (2010), 373-378.

[31] C. J. ZHAO AND W. S. CheUnG, Radial Blaschke-Minkowski homomorphisms and volume differences, Geom. Dedicata 154 (2011), 81-91.

[32] C. J. Zhao, G. S. Leng, Brunn-Minkowski inequality for mixed projection bodies, J. Math. Anal. Appl. 301 (2005), 115-123. 